\title{
MODELACIÓN DE LA EVAPOTRANSPIRACIÓN POTENCIAL EN EL ALTIPLANO DE PUNO
}

\author{
PMODELING OF POTENTIAL EVAPOTRANSPIRATION IN THE HIGH \\ PLAINS OF PUNO
}

\author{
${ }^{1}$ Eduardo Luis Flores Quispe, ${ }^{2}$ Carlos Alberto Silva Delgado, ${ }^{3}$ Eduardo Flores Condori
}

\begin{abstract}
RESUMEN
El objetivo de este trabajo fue realizar la modelación de la evapotranspiración potencial (ETP) en el Altiplano de Puno, en función del rango de temperatura, aplicando la estimación de parámetros de regresión por máxima verosimilitud. Se desarrollaron tres modelos de ETP a nivel mensual para tres regiones homogéneas, se aplicó un método de regionalización por ajuste lineal simple de la tendencia de la ETP mensual. La modelación en cada región homogénea se realizó con un ajuste de la ETP mensual (obtenida por el método del tanque clase A de la publicación 56 de la FAO) a series de Fourier, estos coeficientes de ajuste se relacionan a través de modelos de regresión lineal múltiple con los coeficientes de ajuste del rango de temperatura a series de Fourier y variables geográficas (latitud longitud y elevación). Se utilizaron datos de 12 estaciones. Se calibró y validó los modelos, el período de validación fue de 5 años. La validación se realizó comparando los valores obtenidos de los modelos desarrollados con los valores de ETP de tanque y los valores obtenidos por los métodos de Hargreaves and Samani (1985), García, Sánchez, and Paredes (2000) ySerruto (1993). Los modelos de ETP obtenidos para las tres regiones homogéneas poseen un buen desempeño respecto a los demás modelos, excepto el modelo de la región 2 que tiene deficiencias en estimar la ETP en zonas cercanas al Lago Titicaca. Los modelos de ETP son aplicables a su respectiva región homogénea, estas regiones se encuentran entre los 3812 y 3970 metros sobre el nivel del mar:
\end{abstract}

Palabras claves: Modelación, Evapotranspiración Potencial, Series de Fourier, Máxima Verosimilitud, Regresión múltiple, Rango de temperatura.

\section{ABSTRACT}

The objective of this project was the modeling of potential evapotranspiration (PET) in the high plains of Puno, as a function of temperature range, applying an estimate of regression parameters for maximum likelihood. Three monthly PET models were developed for three homogeneous regions, and was applied a regionalization method for simple linear adjustment of the monthly PET tendency Modeling in each region was carried out with a Fourier Series adjustment of monthly PET (obtained for Class A tank from Paper 56 of the FAO). These adjustment coefficients are related through multiple linear regression models with Fourier Series adjustment coefficients for temperature range and geographic variables (latitude, longitude and elevation). Data from 12 stations was used. The models were calibrated and validated over a period of 5 years. The validation was done by comparing values obtained from the developed models with tank PET values with those obtained by the methods of), ) and). The PET models obtained for the three homogeneous regions performed well compared to other models, except for the region 2 model which showed deficiencies in estimating PET in areas near Titicaca lake. The PET models are applicable to their respective homogeneous regions which are between 3812 and 3970 meters above sea level.

Keywords: Modeling, Potential Evapotranspiration, Fourier Series, Maximum Likelihood, Multiple Regression, Temperature Range

\section{INTRODUCCIÓN}

En el Perú los estudios de los procesos de evapotranspiración son muy escasos, a pesar de que este interviene directamente en el ciclo hidrológico y por tanto la mayor parte del agua se pierde por este proceso a la atmósfera. El estudio de la evapotranspiración potencial es importante, porque su estimación interviene en el cálculo del requeri- miento de agua de los cultivos, en la planificación del riego y en los estudios hidrológicos. A pesar de la baja disponibilidad de los recursos hídricos en el altiplano peruano, poca investigación se ha llevado adelante relacionada a la determinación de los requerimientos de riego bajo las condiciones prevalecientes de clima y suelo (Michel, 1997). Esto hace difícil el dimensionamiento de los sistemas de riego, lo cual resulta en errores significativos en el cálculo de los requeri-

Doctor en Ciencia, Tecnologiay Medio Ambiente. Docente ordinario de la Facultad de Ingeniería de la Universidad Nacional de Moquegua. "Ingeniero en Informática y Sistemas. Docente ordinario de la Facultad de Ingeniería de la Universidad Nacional de Moquegua. Moquegua-Perú. Doctor en Medio Ambiente y Desarrollo Sostenible. Docente Principal de la Universidad Nacional del Altiplano. Puno-Perú 
Flores, E. et al. Modelación de la evapotranspiración potencial en el Altiplano de Puno.

mientos de agua para esas condiciones, especialmente en el caso de cultivos andinos ya que ellos presentan singulares dinámicas relacionadas al sistema agua-suelo-plantaatmósfera. El problema de la determinación de la evapotranspiración potencial en el Altiplano de Puno merece especial atención, debido a la falta de modelos matemáticos adecuados a las características singulares de la zona. La presencia del Lago Titicaca, crea un medio ambiente diferente a otras zonas del Perú ubicadas a la misma altitud, del mismo modo, zonas más alejadas al lago tienen condiciones meteorológicas diversas que influyen en la evapotranspiración potencial.

El objetivo general de la investigación fue realizar la modelación de la evapotranspiración potencial mediante el uso del método de máxima verosimilitud para establecer los parámetros de una nueva fórmula empírica en función de las variables meteorológicas para las singulares condiciones climáticas del Altiplano de Puno. Los objetivos específicos fueron: (1) Establecer uno o varios modelos que permitan determinar la evapotranspiración potencial en función de valores obtenidos de tanque clase $\mathrm{A}$, métodos validados en anteriores investigaciones y registros de variables meteorológicas para las condiciones climáticas del Altiplano de Puno. (2) Calibrar y validar los modelos de evapotranspiración potencial para las condiciones de clima del altiplano de Puno.

Gurovich (1999) afirma que, el método estándar de determinación de la evapotranspiración, es el evaporimetro de bandeja Standard Clase A, del U.S.W.B. (Oficina de Climatología de EE.UU.); este instrumento permite determinar la evaporación desde una superficie de agua, que es el mejor integrador de los factores climáticos que determinan la evapotranspiración.

La ecuación general para estimar ETp (evapotranspiración potencial) por medio de lecturas de evaporación en una bandeja es:

$$
E T p=K p \times E
$$

Dónde: $\mathrm{ETp}=$ evapotranspiración potencial diaria del área bajo estudio ( $\mathrm{mm} / \mathrm{dí}), \mathrm{Kp}=$ coeficiente de bandeja, que depende de la ubicación de ésta y de las condiciones climáticas, $\mathrm{E}$ = evaporación del tanque o bandeja para el periodo de 24 horas ( $\mathrm{mm} /$ día).

Allen, Pereira, Raes and Smith (1998) afirman que, dependiendo del tipo de tanque, el tamaño y estado de la zona de amortiguamiento de viento (fetch) los coeficientes diferirán. $\mathrm{Al}$ agrandar la zona de amortiguamiento de viento, la mayoría de aire en movimiento sobre el tanque estará en equilibrio con la zona de amortiguamiento. En equilibrio con un gran fetch, el aire contiene más vapor de agua y menos calor en el caso A que en el caso B, según las figuras 1 y 2 .

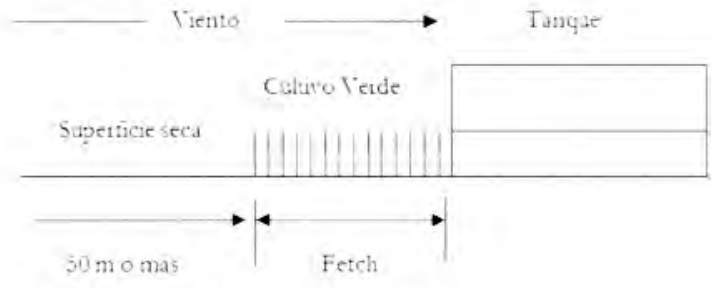

Figura 1. Caso A

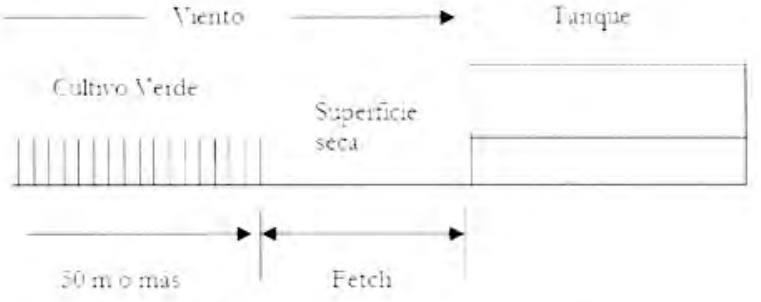

Figura 2. Caso B

La ecuación de regresión para el tanque clase $\mathrm{A}$ y para el caso A, es:

$$
\begin{aligned}
& K p=0,108-0,0286 u_{2}+0,0422 \ln (F E T)+0,1434 \\
& \ln \left(R H_{\max }\right)-0,000631[\ln (F E T)]^{2} \ln \left(R H_{\max }\right)
\end{aligned}
$$

Dónde: $K \phi=$ coeficiente del tanque, $\mathrm{u}_{2}=$ velocidad del viento diaria promedio a $2 \mathrm{~m}$ de altura $(\mathrm{m} / \mathrm{s}), \mathrm{RH}_{\text {mean }}=$ humedad relativa diaria promedio $(\%), \mathrm{FET}=$ borde o fetch. La ecuación anterior es válida dentro de los rangos: $1 \mathrm{~m} \leq$ FET $\leq 1000 \mathrm{~m}, 30 \% \leq \mathrm{RH}_{\operatorname{mex}} \leq 84 \%, 1 \mathrm{~m} / \mathrm{s} \leq \mathrm{u}_{2} \leq 8 \mathrm{~m} / \mathrm{s}$.

Fennessey and Vogel (1996) afirman que, los datos periódicos pueden aproximarse por una función continua de series de Fourier. Bloomfield(1976) reportó la formulación de series de Fourier para aproximar la $\mathrm{E}_{t} \mathrm{O}$. La serie de Fourier aproximada es:

$$
E_{t, f} \approx E_{t a}+\sum_{k=1}^{m}\left[a_{k} \cos \left(\frac{\pi k \tau}{6}\right)+b_{k} \sin \left(\frac{\pi k \tau}{6}\right)\right]
$$

Donde $\tau$ es el mes, $\tau=1, \ldots, 12 ; \mathrm{E}_{t, \mathrm{f}}(\tau)$ denota la aproximación por series de Fourier para el valor promedio mensual de evapotranspiración del cultivo de referencia diaria durante el mes $\tau ; \mathrm{E}_{\mathrm{p}}$ es el promedio anual de evapotranspiración del cultivo de referencia diaria; $\mathrm{k}$ es el índice de sumatoria para el armónico $\mathrm{k}$; $\mathrm{m}$ es el número total de armónicos requeridos para aproximar exactamente $\mathrm{E}_{\mathrm{t}}(\tau)$. Se determinó que con $\mathrm{m}=2$ armónicos resultan valores de $\mathrm{E}_{t, \mathrm{f}}(\tau)$ que aproximan muy bien $\mathrm{E}_{t}(\tau)$. Los coeficientes de la serie de Fourier para el armónico $\mathrm{k}$ de $\mathrm{E}_{\mathrm{t}, \mathrm{f}}(\tau), \mathrm{a}_{\mathrm{k}} \mathrm{y} \mathrm{b}_{\mathrm{k}}$, son estimados usando:

$$
\begin{aligned}
& a_{k}=\frac{1}{6} \sum_{\tau=1}^{12}\left[E_{t}(\tau)-E_{t a}\right] \cos \left(\frac{\pi k \tau}{6}\right) \\
& b_{k}=\frac{1}{6} \sum_{\tau=1}^{12}\left[E_{t}(\tau)-E_{t a}\right] \sin \left(\frac{\pi k \tau}{6}\right)
\end{aligned}
$$

Fennessey and Vogel (1996), en su estudio señalan que, la temperatura del aire cerca de la superficie terrestre es un indicador de la capa límite planetaria de calor, de los flujos de humedad y del balance de energía en la superficie. Por consiguiente se puede estimar $\mathrm{E}_{r}(\tau)$ utilizando una regresión multivariada con la temperatura del aire como importante candidata a variable independiente. Como fue notado por Fennessey and Kirshen (1994), las ecuaciones que describen muchas de las variables individuales de la ecuación de Penman-Monteith son dependientes de la temperatura. La ecuación de regresión regional fue desarrollada tal cual describe cinco coeficientes de Fourier de evapotranspiración del cultivo de referencia de dos armónicos formados: $\mathrm{E}_{\mathrm{a}}, \mathrm{a}_{1}, \mathrm{~b}_{1}$, $a_{2} y_{2}$. Las variables independientes candidatas incluyen la 
Flores, E. et al. Modelación de la evapotranspiración potencial en el Altiplano de Puno.

longitud, elevación, temperatura media mensual y la temperatura media anual.

Fennessey and Vogel (1996), afirman que, las series de Fourier se pueden ajustar a las temperaturas medias mensuales, $\mathrm{T}(\tau), \tau=1, \ldots, 12$, asi:

$$
T_{f}(\tau) \approx T_{a}+\sum_{k=1}^{m}\left[c_{k} \cos \left(\frac{\pi k \tau}{6}\right)+d_{k} \sin \left(\frac{\pi k \tau}{6}\right)\right]
$$

Donde $T_{i}(\tau)$ es la aproximación por series de Fourier de la temperatura promedio mensual diaria $\left(\mathrm{en}^{\circ} \mathrm{C}\right.$ ) para el mes $\tau$ y $T_{a}$ es la temperatura promedio anual diaria. Semejante a $\mathrm{E}_{\mathrm{t}, \mathrm{f}}(\tau)$, se limitó $\mathrm{T}_{\mathrm{f}}(\tau)$ a los primeros dos armónicos, de aquí los cinco coeficientes de las series de Fourier $T_{f}(\tau)$ son descritos por $\mathrm{T}_{2}, \mathrm{c}_{1}, \mathrm{~d}_{1}, \mathrm{c}_{2} \mathrm{y} \mathrm{d}_{2}$ utilizando:

$$
\begin{gathered}
T_{a}=\frac{1}{12} \sum_{\tau=1}^{12} T(\tau) \\
c_{1}=\frac{1}{6} \sum_{\tau=1}^{12}\left[T(\tau)-T_{a}\right] \cos \left(\frac{\pi \tau}{6}\right) \\
d_{1}=\frac{1}{6} \sum_{\tau=1}^{12}\left[T(\tau)-T_{a}\right] \sin \left(\frac{\pi \tau}{6}\right) \\
c_{2}=\frac{1}{6} \sum_{\tau=1}^{12}\left[T(\tau)-T_{a}\right] \cos \left(\frac{\pi \tau}{3}\right) \\
d_{2}=\frac{1}{6} \sum_{\tau=1}^{12}\left[T(\tau)-T_{a}\right] \sin \left(\frac{\pi \tau}{3}\right)
\end{gathered}
$$

Fennessey and Vogel (1996), realizaron el ajuste a ecuaciones para las variables dependientes $E_{t a}, a_{1}, a_{2}, b_{1}$ y $b_{2}$ utilizando las variables independientes: longitud, elevación de la estación, $\mathrm{T}_{\mathrm{a}}, \mathrm{c}_{1}, \mathrm{c}_{2}, \mathrm{~d}_{1} \mathrm{y} \mathrm{d}_{2}$. La ecuación de regresión final toma la forma

$\theta_{t}=e_{0}+e_{1}$ Long $+e_{2}$ Elev $+e_{3} T_{a}+e_{4} c_{1}+e_{5} d_{1}+e_{6} c_{2}+e_{7} d_{2}$

Donde Long es la longitud del sitio (en grados decimales), Elev es la elevación del sitio (en metros), y $\theta_{\mathrm{t}}$ denota la variable dependiente $E_{t}, a_{1}, b_{1}, a_{2} \circ b_{2}$.

El modelo regional final para $\mathrm{E}_{t}(\tau)$, denotado por $E_{t, r}(\tau)$, es:

$$
E_{t, r}(\tau)=E_{i a}+a_{1} \cos \left(\frac{\pi \tau}{6}\right)+b_{1} \sin \left(\frac{\pi \tau}{6}\right)+a_{2} \cos \left(\frac{\pi \tau}{3}\right)+b_{2} \sin \left(\frac{\pi \tau}{3}\right)
$$

Donde los coeficientes del modelo son obtenidos utilizando la ecuación de regresión regional.

Pindyck and Rubinfeld(1998), señalan que la estimación de mínimos cuadrados ordinarios produce estimadores que en ocasiones son ineficientes o inconsistentes. La gran ventaja de la estimación por máxima verosimilitud es que bajo un conjunto amplio de condiciones los estimadores de los parámetros son consistentes y (para muestras grandes) asintóticamente eficientes.

Supóngase que un modelo general está dado por:

$$
Y=f\left(X_{1}, \ldots, X_{k}, \beta_{1}, \ldots, \beta_{p}\right)+\varepsilon
$$

Donde $\varepsilon$ está distribuido en forma normal y satisface todas las otras suposiciones del modelo de regresión lineal básico. Entonces, para cada una de las $\mathrm{N}$ observaciones en $\mathrm{Y}$, y las $\mathrm{X}$ correspondientes, se puede escribir la distribución de probabilidad de $\mathrm{Y}$, dadas las $\mathrm{X}$ y $\beta$, como:

$$
f\left(Y_{i}, X_{i}, \beta\right)=\left[\frac{1}{2 \pi \sigma^{2}}\right]^{1 / 2} \exp \left[\left(-\frac{1}{2 \sigma^{2}}\right)\left(Y_{i}-f\left(X_{1 i}, \ldots, X_{k i}, \beta_{1}, \ldots, \beta_{p}\right)\right)\right]^{2}
$$

Donde exp representa la función exponencial. Entonces, la función log-verosimilitud para todas las $\mathrm{N}$ observaciones está dada por:

$$
\begin{aligned}
\log L= & \sum f\left(Y_{i}, X_{i}, \beta\right)=-\left(\frac{N}{2}\right) \log 2 \pi-\left(\frac{N}{2}\right) \log \sigma^{2} \\
& -\left(\frac{1}{2 \sigma^{2}}\right) \sum\left(Y_{i}-f\left(X_{1 i}, \ldots, X_{k i}, \beta_{1}, \ldots, \beta_{p}\right)\right)
\end{aligned}
$$

(Todas las sumatorias son calculadas sobre las observaciones $\mathrm{i}=1,2, \ldots, \mathrm{N}$.)

$\mathrm{Al}$ diferenciar la última ecuación con respecto a cada una de las $\beta$ y $\sigma^{2}$, igualando a 0 y resolviendo, obtendremos un sistema de $\mathrm{p}+1$ ecuaciones no lineales con $\mathrm{p}+1$ incógnitas. Si las ecuaciones no son lineales, el proceso de solución es más complejo y se debe usar un procedimiento numérico.

Algunos autores obtuvieron ecuaciones de ETP adecuadas para el altiplano de Puno. Según García et al.(2000), el modelo de mejor ajuste de ETP corresponde al potencial cuya expresión es para Puno.

$$
E_{0}=3,2114\left(Q_{s}\right)^{0,005627 \Delta \mathrm{T}}
$$

Dónde: $E_{o}=$ evapotranspiración potencial $(\mathrm{mm} /$ día $), Q^{s}=$ radiación solar extraterrestre $(\mathrm{mm} /$ día $)$, $\Delta T=$ rango diurno de temperatura $\left({ }^{\circ} \mathrm{C}\right), \Delta T=T$ max $-T$ min . Serruto (1993) propone el modelo siguiente:

$$
E T P=0,003(R S)^{2,5}+0,16(T)^{0,88}
$$

Dónde: ETP = evapotranspiración potencial o evapotranspiración de referencia $(\mathrm{mm} /$ día $), R S=$ radiación solar extraterrestre $(\mathrm{mm} /$ día), $\mathrm{T}=$ temperatura media mensual $\left({ }^{\circ} \mathrm{C}\right)$.

Allen et al. (1998) plantean, que la ETo puede ser estimada utilizando la ecuación de Hargreaves and Samani (1985) donde:

$$
E T_{0}=0,023\left(T_{\text {maxt }}+17,8\right)\left(T_{\max }-T_{\text {man }}\right){ }^{0.5} \mathrm{R} a
$$

Dónde: $E T_{0}=$ evapotranspiración del cultivo de referencia $(\mathrm{mm} /$ día $), T_{\text {man }}=$ temperatura promedio del día $\left({ }^{\circ} \mathrm{C}\right), T_{\max }=$ temperatura máxima promedio del día $\left({ }^{\circ} \mathrm{C}\right), T_{\min }$ $=$ temperatura mínima promedio del dia $\left({ }^{\circ} \mathrm{C}\right), \mathrm{Ra}=$ radiación solar extraterrestre ( $\mathrm{mm} / \mathrm{día})$.

\section{MATERIALES Y MÉTODOS}

Se utilizó registros meteorológicos de 20 años en promedio, de 12 estaciones, obtenidas del Servicio Nacional de Meteorología e Hidrología (Senamhi), consistentes en: Temperatura media de las máximas y de las minimas, Humedad relativa media mensual, Velocidad del viento, Evaporación mensual de tanque clase " $\mathrm{A}$ ".

Las estaciones meteorológicas fueron las siguientes que se muestran en la tabla 1 . 
Flores, E. et al. Modelación de la evapotranspiración potencial en el Altiplano de Puno.

Tabla 1. Estaciones meteorológicas consideradas.

\begin{tabular}{lccc}
\hline Estación & Longitud (W) & Latitud (S) & Altitud (msnm) \\
\hline Arapa & $70^{\circ} 06^{\prime} 00^{\prime \prime}$ & $15^{\circ} 09^{\prime} 00^{\prime \prime}$ & 3840 \\
Ayaviri & $70^{\circ} 35^{\prime} 35^{\prime \prime}$ & $14^{\circ} 52^{\prime} 23^{\prime \prime}$ & 3928 \\
Azángaro & $70^{\circ} 12^{\prime} 00^{\prime \prime}$ & $14^{\circ} 55^{\prime} 00^{\prime \prime}$ & 3860 \\
Desaguadero & $69^{\circ} 02^{\prime} 00^{\prime \prime}$ & $16^{\circ} 34^{\prime} 00^{\prime \prime}$ & 3812 \\
El Belén & $68^{\circ} 40^{\prime} 00^{\prime \prime}$ & $16^{\circ} 04^{\prime} 00^{\prime \prime}$ & 3820 \\
Huancané & $69^{\circ} 45^{\prime} 00^{\prime \prime}$ & $15^{\circ} 12^{\prime} 00^{\prime \prime}$ & 3890 \\
Huaraya Moho & $69^{\circ} 28^{\prime} 00^{\prime \prime}$ & $15^{\circ} 23^{\prime} 00^{\prime \prime}$ & 3890 \\
Juli & $69^{\circ} 27^{\prime} 00^{\prime \prime}$ & $16^{\circ} 13^{\prime} 00^{\prime \prime}$ & 3820 \\
Lampa & $70^{\circ} 22^{\prime} 00^{\prime \prime}$ & $15^{\circ} 22^{\prime} 00^{\prime \prime}$ & 3892 \\
Melgar & $70^{\circ} 54^{\prime} 03^{\prime \prime}$ & $14^{\circ} 46^{\prime} 06^{\prime \prime}$ & 3920 \\
Progreso & $70^{\circ} 21^{\prime} 57^{\prime \prime}$ & $14^{\circ} 41^{\prime} 22^{\prime \prime}$ & 3970 \\
Puno & $70^{\circ} 01^{\prime} 00^{\prime \prime}$ & $15^{\circ} 50^{\prime} 00^{\prime \prime}$ & 3812 \\
\hline
\end{tabular}

\section{Metodología}

La evapotranspiración potencial (ETP) se determinó por el método del tanque clase "A" del Paper 56 de la FAO, esta se consideró como ETP observada.

El coeficiente del tanque clase A se determinó según el procedimiento citado por Allen et al. (1998). El caso que se consideró fue A mostrado en la figura 1, condición similar en la que operan los tanques en el Altiplano de Puno. Se utilizó la ecuación 2 del coeficiente para el tanque clase $A$.

La ecuación de regresión lineal simple de la evapotranspiración media mensual contra el tiempo (año) en los últimos 5 años de cada estación, se estimó por mínimos cuadrados y los parámetros de esta ecuación (intercepto y pendiente) se tomaron para definir regiones homogéneas. Para ello se graficó cada parámetro (intercepto y/o pendiente) contra la Longitud geográfica en grados decimales. Donde se observó tendencias lineales diferentes entre los puntos, se encontró una región homogénea en evapotranspiración potencial.

La serie de Fourier a la que se ajustó la ETP mensual es la que presenta la ecuación 3 .

Los coeficientes de la serie de Fourier para el armónico $k$ de $\mathrm{E}_{\mathrm{t}, \mathrm{i}}(\tau), \mathrm{a}_{\mathrm{k}} \mathrm{y} \mathrm{b}_{\mathrm{k}}$, fueron estimados usando las ecuaciones 4 y 5 .

El rango de temperatura Dt (diferencia entre la temperatura media de las máximas y la media de las mínimas) del aire es un indicador del nivel de evapotranspiración sobre la superficie terrestre. La ETP mensual se estima utilizando una regresión multivariada con el rango de temperatura como variable independiente.

La ecuación de regresión regional describe cinco coeficientes de Fourier de ETP formado por dos armónicos: $\mathrm{E}_{\mathrm{b}}, \mathrm{a}_{1}, \mathrm{~b}_{1}, \mathrm{a}_{2} \mathrm{y} \mathrm{b}_{2}$. Las variables independientes son: la latitud, la longitud y la elevación de la estación, y el rango de temperatura promedio.

Las series de Fourier fueron ajustadas a los rangos de temperatura mensuales promedio, $\operatorname{Dt}(\tau), \tau=1, \ldots, 12$, mostrada por:

$$
D t_{f}(\tau) \approx D t_{a}+\sum_{k=1}^{m}\left[c_{k} \cos \left(\frac{\pi k \tau}{6}\right)+d_{k} \sin \left(\frac{\pi k \tau}{6}\right)\right]
$$

Donde $\mathrm{Dt}_{\mathrm{f}}(\tau)$ es la aproximación por series de Fourier del rango de temperatura mensual diaria $\left(\mathrm{en}^{\circ} \mathrm{C}\right)$ para el mes $\tau$ y $\mathrm{Dt}_{\mathrm{a}}$ es el rango de temperatura promedio anual. Semejante a $\mathrm{E}_{t, f}(\tau)$, se limitó $\mathrm{Dt}_{i}(\tau)$ a los primeros dos armónicos, los 5 coeficientes de la serie de Fourier $\mathrm{Dt}_{\mathrm{p}}(\tau)$ son descritos por $\mathrm{Dt}_{\mathrm{a}}, \mathrm{c}_{1}, \mathrm{~d}_{1}, \mathrm{c}_{2} \mathrm{y} \mathrm{d}_{2}$ utilizando:

$$
\begin{gathered}
D t_{a}=\frac{1}{12} \sum_{\tau=1}^{12} D t(\tau) \\
c_{1}=\frac{1}{6} \sum_{\tau=1}^{12}\left[D t(\tau)-D t_{a}\right] \cos \left(\frac{\pi \tau}{6}\right) \\
d_{1}=\frac{1}{6} \sum_{\tau=1}^{12}\left[D t(\tau)-D t_{a}\right] \sin \left(\frac{\pi \tau}{6}\right) \\
c_{2}=\frac{1}{6} \sum_{\tau=1}^{12}\left[D t(\tau)-D t_{a}\right] \cos \left(\frac{\pi \tau}{3}\right) \\
d_{2}=\frac{1}{6} \sum_{\tau=1}^{12}\left[D t(\tau)-D t_{a}\right] \sin \left(\frac{\pi \tau}{3}\right)
\end{gathered}
$$

Dónde: $\operatorname{Dt}(\tau)=$ rango de temperatura mensual promedio $\left({ }^{\circ} \mathrm{C}\right)$ del mes $\tau, \mathrm{Dt}_{\mathrm{a}}=$ rango de temperatura anual promedio $\left({ }^{\circ} \mathrm{C}\right)$.

La ecuación de regresión regional final toma la forma:

$$
\theta_{t}=e_{0}+e_{1} L a t+e_{2} L \text { ong }+e_{3} \text { Elev }+e_{4} D t_{a}+e_{5} c_{1}+e_{6} d_{1}+e_{7} c_{2}+e_{8} d_{2}(20)
$$

Donde Lat es la latitud, Long es la longitud (ambos en grados decimales), Elev es la elevación del sitio (en metros sobre el nivel del mar), y $\theta_{t}$ denota la variable dependiente $\mathrm{E}_{\mathrm{ta}}$, $a_{1}, b_{1}, a_{2} \circ b_{2}$.

El modelo regional final para $\mathrm{E}_{1}(\tau)$, denotado por $\mathrm{E}_{\mathrm{tr}}(\tau), \mathrm{es}:$

$$
\begin{aligned}
E_{t, r}(\tau)= & E_{t a}+a_{1} \cos \left(\frac{\pi \tau}{6}\right)+b_{1} \sin \left(\frac{\pi \tau}{6}\right) \\
& +a_{2} \cos \left(\frac{\pi \tau}{3}\right)+b_{2} \sin \left(\frac{\pi \tau}{3}\right)
\end{aligned}
$$

Donde los coeficientes del modelo son obtenidos utilizando la ecuación de regresión regional.

Se estimó los coeficientes de la ecuación de regresión por el método de máxima verosimilitud. Para la estimación por máxima verosimilitud se utilizó el software Eviews versión 5.0.

El período de calibración se tomó en cada estación como toda la serie de registro excepto los últimos cinco años que se utilizaron para la validación del modelo.

La calibración del modelo se realizó con la estimación de los coeficientes de regresión múltiple primero por el método de mínimos cuadrados y posteriormente por el método de máxima verosimilitud.

La validación del modelo se realizó con la aplicación del modelo a este período y se comparó los valores calculados con los observados. Simultáneamente se comparó los valores obtenidos por series de Fourier con los obtenidos aplicando los modelos desarrollados en el Altiplano (Serruto, 1993) (García et al., 2000) y con el modelo de Hargreaves and Samani (1985). Para la comparación del modelo se determinó el error medio cuadrático (EMC). 
Flores, E. et al. Modelación de la evapotranspiración potencial en el Altiplano de Puno.

\section{RESULTADOSY DISCUSIÓN}

La figura 3 muestra la tendencia de la ETP mensual contra el tiempo para la estación Desaguadero, este mismo análisis se realizó para las 12 estaciones.

Gráico EIP vs Tiempo. Estaciòn Desaguadero

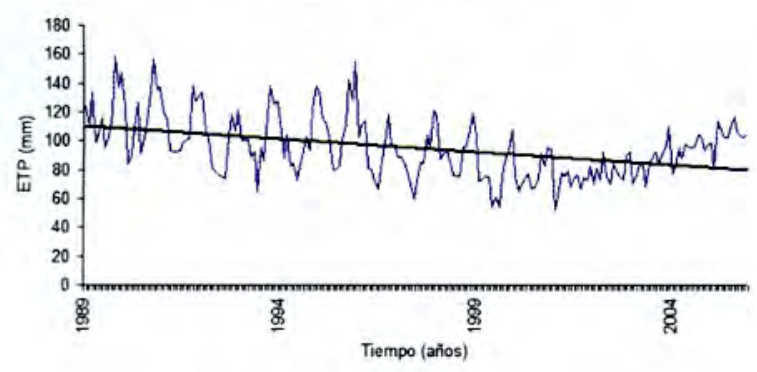

Figura 3. ETP mensual contra el tiempo. Estación Desaguadero

El ajuste lineal de la ETP media mensual de los últimos 5 años contra el tiempo, para la estación Desaguadero se muestra en la figura 4, para las 12 estaciones también se hizo este ajuste.

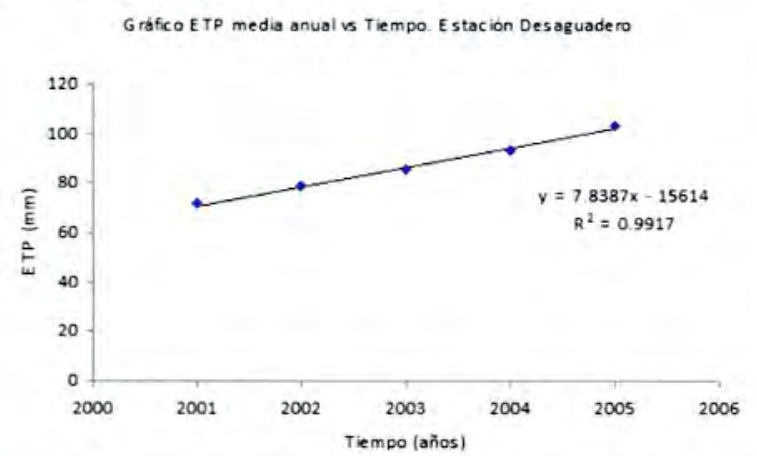

Figura 4. ETP media mensual contra el tiempo. Estación Desaguadero

La figura 5 de la pendiente de la línea de ajuste contra la longitud geográfica determina patrones lineales diferentes que siguen las estaciones que pertenecen a una región homogénea, se presenta los valores de longitud en cada punto. En esta figura claramente se observa tres regiones homogéneas en ETP.

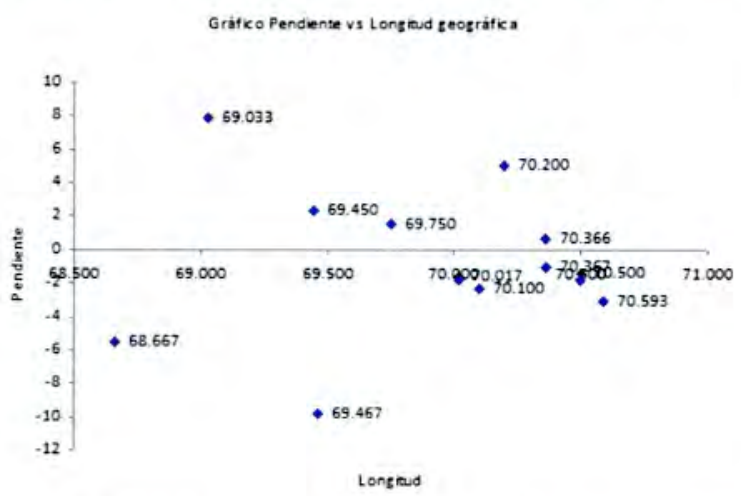

Figura 5. Pendiente contra la longitud geográfica de las Estaciones
En la tabla 2 se muestra las tres regiones homogéneas en ETP, según los patrones observados con la pendiente y la longitud.

Tabla 2. Región homogénea 1 según la pendiente y la longitud

\begin{tabular}{lrlll}
\hline Región 1 & \multicolumn{4}{c}{ Pendiente Longitud (W) Latitud (S) Altitud (msnm) } \\
El Belen & $-5,538$ & 68,667 & 16,067 & 3820 \\
Huaraya Moho & $-9,813$ & 69,467 & 15,383 & 3890 \\
\hline
\end{tabular}

Tabla 3. Región homogénea 2 según la pendiente y la longitud

\begin{tabular}{|c|c|c|c|c|}
\hline Región 2 & Pendiente & Longitud (W) & Latitud (S) & Altitud (msnm) \\
\hline Desaguadero & 7,838 & 69,033 & 16,567 & 3812 \\
\hline Juli & 2,308 & 69,450 & 16,217 & 3820 \\
\hline Huancane & 1,473 & 69,750 & 15,200 & 3890 \\
\hline Puno & $-1,607$ & 70,017 & 15,833 & 3812 \\
\hline Arapa & $-2,385$ & 70,100 & 15,150 & 3840 \\
\hline
\end{tabular}

Tabla 4. Región homogénea 3 según la pendiente y la longitud

\begin{tabular}{lclll}
\hline Región 3 & \multicolumn{2}{c}{ Pendiente Longitud (W) } & Latitud (S) & Altitud (msnm) \\
\hline Azangaro & 5,055 & 70,200 & 14,917 & 3860 \\
Progreso & 0,6 & 70,366 & 14,689 & 3970 \\
Lampa & $-1,02$ & 70,367 & 15,367 & 3892 \\
Melgar & $-1,422$ & 70,500 & 14,768 & 3920 \\
Ayaviri & $-3,068$ & 70,593 & 14,873 & 3928 \\
\hline
\end{tabular}

En la figura 6 se muestra las regiones homogéneas en

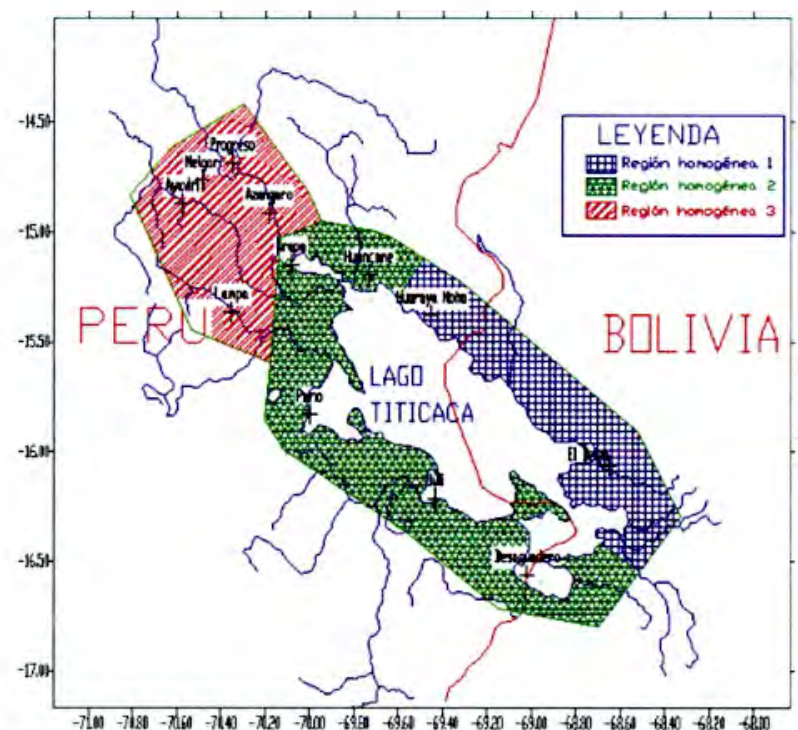

Figura 6. Ubicación de las regiones homogéneas en ETP

En la tabla 5 se muestra el periodo de calibración para las tres regiones homogéneas.

En la tabla 6 se muestran los resultados del proceso de calibración del modelo para cada región homogénea a través del método de mínimos cuadrados, se presentan los coeficientes de determinación $\mathrm{R}^{2} \mathrm{y}$ de correlación múltiple $\mathrm{R}$.

El modelo de ETP que se calibró para cada región homogénea tiene un buen desempeño en las regiones 1 y 3 esto se muestra con altos valores de coeficientes de determinación y correlación. En cambio el desempeño del modelo en la región 2 es deficiente. 


\section{Ciencia \& Desarrollo}

Flores, E. et al. Modelación de la evapotranspiración potencial en el Altiplano de Puno

Tabla 5. Período de calibración del modelo para las regiones homogéneas.

\begin{tabular}{ccl}
\hline Región Homogénea & Estación & Periodo de calibración \\
\hline 1 & El Belen & $1991-1995$ \\
& Huaraya Moho & $1985-1995$ \\
2 & Arapa & $1964-1970$ \\
& Desaguadero & $1989-2000$ \\
Huancané & $1992-2000$ \\
& juli & $1991-2000$ \\
& Puno & $1981-2000$ \\
3 & Ayaviri & $2002-2006$ \\
& Azángaro & $1964-1966$ \\
& Lampa & $1996-2001$ \\
& Melgar & $2000-2004$ \\
& Progreso & $1970-1978$ \\
\hline
\end{tabular}

Tabla 6. Resultados del proceso de calibración de los modelos de ETP en las regiones homogéneas.

\begin{tabular}{|c|c|c|c|}
\hline Región homogénea & Variable dependiente & $\mathbf{R}^{2}$ & $\mathbf{R}$ \\
\hline \multirow[t]{5}{*}{1} & Eta & 0,91 & 0,96 \\
\hline & $a_{1}$ & 0,74 & 0,86 \\
\hline & $b_{1}$ & 0,42 & 0,65 \\
\hline & $\mathrm{a}_{2}$ & 0,46 & 0,68 \\
\hline & $\mathrm{b}_{2}$ & 0,31 & 0,56 \\
\hline \multirow[t]{5}{*}{2} & Eta & 0,54 & 0,73 \\
\hline & $a_{i}$ & 0,50 & 0,71 \\
\hline & $b_{1}$ & 0,52 & 0,72 \\
\hline & $a_{2}$ & 0,32 & 0,57 \\
\hline & $\mathrm{b}_{2}$ & 0,34 & 0,58 \\
\hline \multirow[t]{5}{*}{3} & Eta & 0,91 & 0,95 \\
\hline & $a_{1}$ & 0,61 & 0,78 \\
\hline & $b_{i}$ & 0,79 & 0,89 \\
\hline & $a_{1}$ & 0,55 & 0,74 \\
\hline & $b_{2}$ & 0,75 & 0,87 \\
\hline
\end{tabular}

Tabla 7. Período de validación del modelo en las regiones homogéneas

\begin{tabular}{ccc}
\hline Región homogénea & Estación & Periodo de validación \\
\hline 1 & El Belen & $1996-2000$ \\
& Huaraya Moho & $1996-2000$ \\
2 & Arapa & $1971-1975$ \\
Desaguadero & $2001-2005$ \\
& Huancané & $2001-2005$ \\
Juli & $2001-2005$ \\
Puno & $2001-2005$ \\
3 & Lampa & $2002-2006$ \\
& Melgar & $2005-2006$ \\
& Progreso & $1979-1983$ \\
\end{tabular}

En la región 2 cercana al Lago en la parte occidental, la ETP no solo depende de la temperatura y de los factores geográficos, sino existe influencia del Lago. Esto se puede afirmar porque el mejor desempeño del modelo se da en la región 3 que está más lejana al Lago.

Los coeficientes de regresión múltiple se estimaron por máxima verosimilitud, estos son los que se presentan finalmente en los modelos de las 3 regiones homogéneas y fueron utilizados para validar los modelos.

El periodo de validación se muestra en la tabla 7 , para las estaciones en que se validaron el modelo.

\section{Región bomogénea 1}

En la estación el Belen el modelo de ETP que se desarrolló tiene un mejor desempeño (o ajuste) que los otros métodos debido a que presenta el menor error medio cuadrático, esto también se puede observar en la figura 7. En esta región el modelo tiene mejor desempeño que los otros métodos.

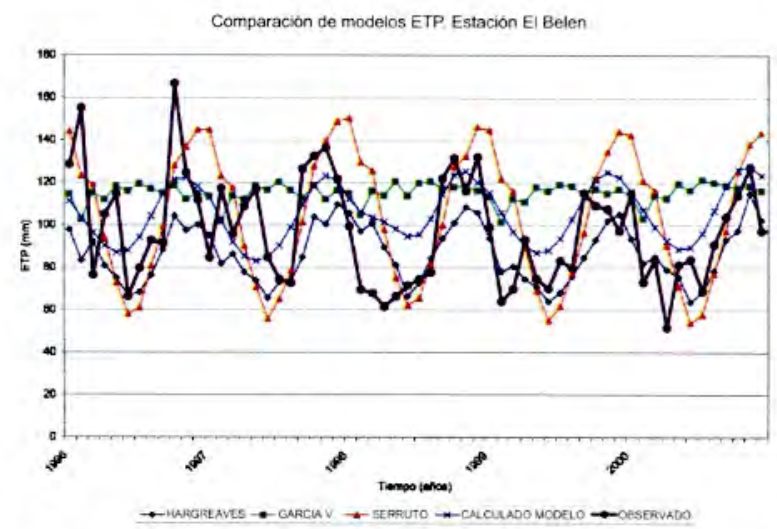

Figura 7. Comparación de modelos de ETP en el periodo de validación. Estación El Beien.

\section{Región homogénea 2}

En esta región homogénea el desempeño del modelo es moderadamente eficiente en estaciones lejanas al Lago.

En las figuras 8 y 9 se muestra el desempeño del modelo en la estación en la que existe el mejor y el peor ajuste, respectivamente a los valores observados.

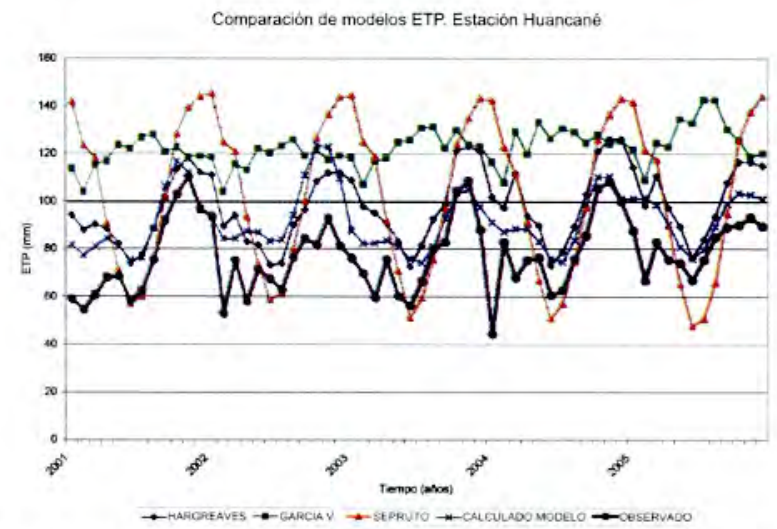

Figura 8. Comparación de modelos de ETP en el período de validación. Estación Huancané

El modelo propuesto según la figura 8 en la estación Huancané, es el que presenta un mejor ajuste a los datos observados, esto se confirma por presentar también el menor error medio cuadrático.

Esta figura 9 muestra que para la estación Puno, el modelo de Hargreaves es el que mejor se ajusta a los datos observados, tiene un error medio cuadrático menor a los demás modelos. El modelo propuesto es poco eficiente en esta estación debido principalmente a la cercanía al Lago y a su efecto en la ETP que no es captado por el modelo desarrollado lo mismo que en la estación Desaguadero y Juli. 
Flores, E. et al. Modelación de la evapotranspiración potencial en el Altiplano de Puno.

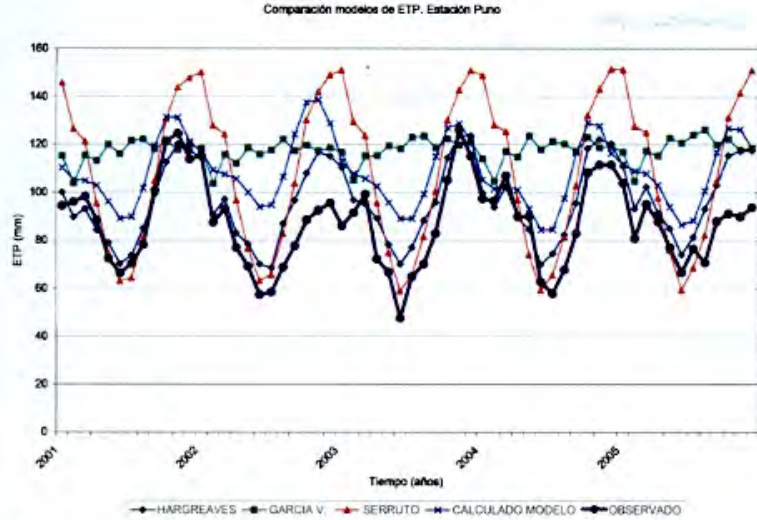

Figura 9. Comparación de modelos de ETP en el período de validación. Estación Puno

\section{Región bomogénea 3}

Según la figura 10, el modelo propuesto en la estación Lampa, tiene un buen ajuste a los datos observados que los demás modelos, tiene también el menor error medio cuadrático. El desempeño del modelo en las estaciones de esta región homogénea es muy prometedor.

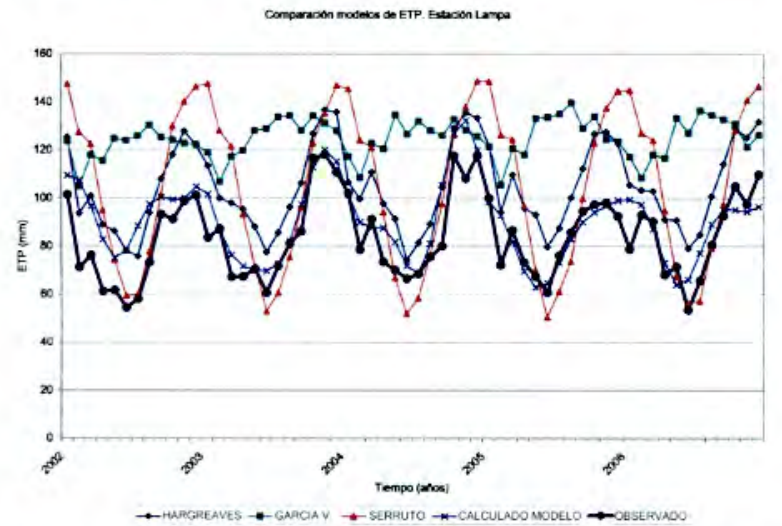

Figura 10. Comparación de modelos de ETP en el periodo de validación. Estación Lampa

En las tablas siguientes, se muestra los errores medios cuadráticos (EMC) obtenido en el período de validación. Según estas tablas cada modelo propuesto para cada región homogénea presenta un buen desempeño (menor EMC) en la mayoría de las estaciones.

Tabla 8. Error medio cuadrático $\left(\mathrm{mm}^{2}\right)$ de los modelos comparados con el modelo propuesto. Región homogénea 1.

\begin{tabular}{cllll}
\hline Estación & \multicolumn{3}{l}{ Hargreaves Garcia V, Serruto Modelo propuesto } \\
\hline El Belen & 544,6 & 981,2 & 847,7 & 497,6 \\
Huaraya Moho & 420,4 & 1609,2 & 1166,7 & 249,2 \\
\hline
\end{tabular}

Tabla 9. Error medio cuadrático $\left(\mathrm{mm}^{2}\right)$ de los modelos comparados con el modelo propuesto. Región homogénea 2.

\begin{tabular}{cllll}
\hline Estación & Hargreaves & García V, & Serruto Modelo propuesto \\
\hline Arapa & 1068,0 & 2448,0 & 2787,7 & 188,1 \\
Desaguadero & 536,5 & 1273,5 & 1678,7 & 662,9 \\
Huancané & 507,4 & 2269,2 & 1445,3 & 305,4 \\
Juil & 139,1 & 889,4 & 631,2 & 645,1 \\
Puno & 154,1 & 1276,5 & 820,0 & 601,1 \\
\hline
\end{tabular}

Tabla 10. Error medio cuadrático $\left(\mathrm{mm}^{2}\right)$ de los modelos comparados con el modelo propuesto. Región homogénea 3.

\begin{tabular}{ccccc}
\hline Estación & Hargreaves & García V. & Serruto & Modelo propuesto \\
\hline Lampa & 463,7 & 2028,8 & 953,7 & 148,6 \\
Melgar & 863,1 & 2448,6 & 2321,7 & 95,5 \\
Progreso & 1344,1 & 160,8 & 1683,9 & 441,9 \\
\hline
\end{tabular}

El modelo para estimar la evapotranspiración potencial para las regiones homogéneas son

$$
\begin{aligned}
E_{t, r}(\tau)= & E_{t a}+a_{1} \cos \left(\frac{\pi \tau}{6}\right)+b_{1} \sin \left(\frac{\pi \tau}{6}\right) \\
& +a_{2} \cos \left(\frac{\pi \tau}{3}\right)+b_{2} \sin \left(\frac{\pi \tau}{3}\right)
\end{aligned}
$$

Dónde: $\mathrm{E}_{\mathrm{t}, \mathrm{f}}(\tau)=$ evapotranspiración potencial en mm del mes $\tau, \tau=$ indice de mes $1,2, \ldots, 12$. Eta, $a_{1}, b_{1}, a_{2} y b_{2}$ se obtienen de 5 ecuaciones de regresión lineal múltiple del tipo:

$$
\theta_{i}=e_{0}+e_{1} L a t+e_{2} L \text { ong }+e_{3} E l e v+e_{4} D t_{s}+e_{5} c_{1}+e_{6} d_{1}+e_{-} c_{2}+e_{8} d_{2}
$$

Los coeficientes de estas ecuaciones obtenidos por máxima verosimilitud son los que se muestran en las tablas siguientes.

Tabla 11. Coeficientes de regresión lineal múltiple para la región 1 .

\begin{tabular}{cccccccccc} 
日i & e0 & e1 & e2 & e3 & e4 & e5 & e6 & e7 & e8 \\
\hline Eta & 922,7406 & 0 & 0 & $-0,2127$ & $-0,5728$ & 1,5673 & $-4,3370$ & $-4,6834$ & $-5,7186$ \\
a1 & 924,1884 & 0 & 0 & $-0,2436$ & 1,0754 & $-3,5857$ & $-0,3201$ & 4,6650 & 1,1811 \\
b1 & $-640,8246$ & 0 & 0 & 0,1673 & 0,7534 & 6,8575 & 2,1134 & 1,2528 & $-1,6533$ \\
a2 & $-103,2447$ & 0 & 0 & 0,0271 & $-0,1276$ & 0,3642 & 1,3030 & 2,4540 & $-4,6338$ \\
b2 & 242,5945 & 0 & 0 & $-0,0612$ & $-1,7685$ & $-3,9872$ & $-1,4792$ & $-0,2360$ & 0,8854 \\
\hline
\end{tabular}

\begin{tabular}{|c|c|c|c|c|c|c|c|c|c|}
\hline 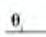 & $c_{5}$ & $e_{1}$ & $\mathrm{e}_{1}$ & $e_{1}$ & $c_{4}$ & $c_{1}$ & $c_{4}$ & $c_{2}$ & c, \\
\hline & $-7383,3610$ & 72,5293 & 55,8543 & 0.6429 & $-0,06773$ & 2,0407 & 5,0163 & $-3,3832$ & 5,94 \\
\hline & -366 & 38,9 & & דים & 3 & 75 & 2.2825 & 1 & 150 \\
\hline & .434 & $3 x$ & 35,7361 & & & & & & 0.57 \\
\hline & 2666,240 & $.21,651$ & 2) & 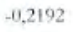 & & 2,2286 & 0,4971 & $1,7=0$ & \\
\hline & 20194,2260 & $-18,5449$ & $-12,3757$ & $-0,2516$ & 1,4569 & 0,0823 & 0,1593 & 1,1357 & 2,16 \\
\hline
\end{tabular}

Tabla 12. Coeficientes de regresión lineal múltiple para la región 2 .

\begin{tabular}{|c|c|c|c|c|c|c|c|c|c|}
\hline 0. & $c_{4}$ & $c_{1}$ & $c_{2}$ & $c_{3}$ & $\mathrm{e}_{4}$ & $e_{s}$ & e, & $c_{\text {. }}$ & $s_{3}$ \\
\hline Eta & 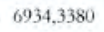 & & 364 & 0,20009 & 5,3529 & 6,4978 & 5,2698 & $-4,2250$ & ובסהי, \\
\hline 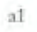 & & & & 0,0154 & $-0,6845$ & $-0,2023$ & $-1,6305$ & 2,0368 & \\
\hline b1 & -506 & & 54 & 0,1 & $-0,5230$ & $-1,6831$ & 2,5868 & 0,9 & 4,0 \\
\hline a2 & & & & 0 & -0.0 & 0,8240 & $-4,7544$ & 6,2964 & 1 , \\
\hline & 2540 & 3,747 & 3,563 & 134 & 1,0825 & 5467 & 0,5034 & 0,374 & 10,11 \\
\hline
\end{tabular}

Tabla 13. Coeficientes de regresión lineal múltiple para la región 3 .

Dónde: $\theta_{j}=$ variable dependiente, sea Et $a, a 1, b 1, a 2$ o $b 2, L a t=$ latitud geográfica en grados decimales, Long = longitud geográfica en grados decimales, Elev = elevación en metros sobre el nivel del mar, Dta, $c 1, d 1, c 2$ y $d 2$, se obtienen a través de las siguientes fórmulas:

$$
D t_{a}=\frac{1}{12} \sum_{\tau=1}^{12} D t(\tau)
$$


Flores, E. et al. Modelación de la evapotranspiración potencial en el Altiplano de Puno.

$$
\begin{aligned}
& c_{1}=\frac{1}{6} \sum_{\tau=1}^{12}\left[D t(\tau)-D t_{a}\right] \cos \left(\frac{\pi \tau}{6}\right) \\
& d_{1}=\frac{1}{6} \sum_{\tau=1}^{12}\left[D t(\tau)-D t_{a}\right] \sin \left(\frac{\pi \tau}{6}\right) \\
& c_{2}=\frac{1}{6} \sum_{\tau=1}^{12}\left[D t(\tau)-D t_{a}\right] \cos \left(\frac{\pi \tau}{3}\right) \\
& d_{2}=\frac{1}{6} \sum_{\tau=1}^{12}\left[D t(\tau)-D t_{a}\right] \sin \left(\frac{\pi \tau}{3}\right)
\end{aligned}
$$

Dónde: $\tau=$ índice de mes $1,2, \ldots, 12, D t=$ rango de temperatura mensual, se define por:

$$
D t=T \max -T \min
$$

Dónde: Tmax $=$ temperatura media de las máximas diarias $\left({ }^{\circ} \mathrm{C}\right)$ en un mes, Tmin = temperatura media de las mínimas diarias $\left({ }^{\circ} \mathrm{C}\right)$ en un mes, Dta $=$ rango de temperatura promedio anual $\left({ }^{\circ} \mathrm{C}\right)$.

\section{CONCLUSIONES}

Los modelos empíricos de evapotranspiración potencial de series de Fourier propuestos para las tres regiones homogéneas, permiten estimar muy bien los valores de ETP obtenidos por el método del tanque clase A para las regiones homogéneas 1 y 3 . En la región homogénea 2 existe poca eficiencia del modelo para estimar la ETP de tanque clase A debido a su cercanía al Lago. Según esto, el comportamiento de la ETP en esta zona no es suficientemente explicado con los factores geográficos y de rango de temperatura.

Los modelos empíricos de ETP con series de Fourier probaron ser más eficientes en su desempeño para estimar la ETP en las regiones homogéneas 1 y 3 , que otros tipos de modelos matemáticos como los de Hargreaves and Samani (1985), García et al. (2000) y Serruto (1993). En la región homogénea 2 se recomienda el uso del método de Hargreaves and Samani (1985).

Los estimadores de máxima verosimilitud bajo un conjunto amplio de condiciones son consistentes y (para muestras grandes) asintóticamente eficientes.

La validación muestra el buen desempeño de los modelos y una aproximación del fenómeno físico sin haber sido desarrollado basado físicamente, sino desde conceptos empíricos pero basados en evidencia física.

Los tres modelos son adecuados para estimar la ETP cada uno en una región homogénea del altiplano de Puno. La región 1 se encuentra en la parte oriental, la región 2 en la parte occidental, ambas cercanas al Lago. La región 3 se encuentra en la zona norte al Lago alejada de este. El modelo en la región 1 es aplicable entre los 3820 a 3890 m.s.n.m. El modelo en la región 2 es aplicable entre los 3812 a 3890 m.s.n.m. pero presenta deficiencias cerca al Lago. El modelo en la región 3 es aplicable entre los 3860 a 3970 m.s.n.m.

\section{REFERENCIAS BIBLIOGRÁFICAS}

Allen, R. G., Pereira, L. S., Raes, D., \& Smith, M. (1998). Crop evapotranspiration: Guidelines for computing crop water requirements, Irrigation and Drainage Paper 56. Rome, Italy: Food and Agricultural Organization of the United Nations.

Bloomfield, P. (1976). Fourier analysis of time series: An introduction. New York, USA: Wiley.

Fennessey, N. M. \& Kirshen, P. H. (1994). Evaporation and evapotranspiration under climate change in New England. ASCE J. Water Resour. Plann. Manage., 120 (1), 48-69.

Fennessey, N. M. \& Vogel, R. M. (1996). Regional models of potential evaporation and reference evapotranspiration for the northeast USA. Journal of Hydrology, 184(3-4), 337-354. doi: $10.1016 / 0022$ 1694(95)02980-x

Garcia, J., Sánchez, O., \& Paredes, M. (2000). Métodos de estimación de la evapotranspiración potencial en función del rango diurno de temperatura (dt) y la radiación solar extraterrestre (qs). Anales Cientificos, Marzo.

Gurovich, L. A. (1999). Riego superficial tecnificado. México D.F., México: Alfaomega.

Hargreaves, G. H., \& Samani, Z. A. (1985). Reference Crop Evapotranspiration from Temperature. Applied Engineering in Agriculture, 1(2), 96-99.

Michel, T. (1997). Zonificación Agroclimática del Altiplano Paceño. La Paz, Bolivia: UMSA y Servicio Nacional de Meteorología e Hidrología.

Pindyck, R. S. \& Rubinfeld, D. L. (1998). Econometric Models and Economic Forecasts. New York, USA: McGrawHill.

Serruto, R. (1993). Una nueva förmula para el cálculo de la evapotranspiración potencial. Puno, Perú: PIWA.

\section{Correspondencia:}

Eduardo Luis Flores Quispe: edu_fa@hotmail.com
Fecha de Recepción: 05/08/2015

Fecha de Aceptación: 12/11/2015 